\title{
Development of Dynamic Model of a 7DOF Hydraulically Actuated Tele-Operated Robot for Decommissioning Applications
}

\author{
Allahyar Montazeri, Josef Ekotuyo
}

\begin{abstract}
In this paper the problem of system integration and dynamic modeling of a hydraulically actuated manipulator with seven degrees of freedom, i.e. HydroLek HLK-7W is investigated. The arm is fitted on Multi-Arm mobile Robot System for Nuclear Decommissioning (MARS-ND) applications purposes. This is a step forward with respect to the previous works where only kinematics of the robot was taking into account. As the decommissioning robot has to perform precise and complex tasks autonomously using effective model-based nonlinear control algorithms having an accurate dynamic model of the arm which is reliable enough to predict the behavior of the manipulator under different operating conditions would be crucial. To this end the symbolic, and numerical model of the dynamic of robot is developed and a first attempt for model validation and tuning the parameters of the model is taken forward.
\end{abstract}

\section{INTRODUCTION}

Modifications to plant units in nuclear power station, plant faults, and operation errors, have led to a number of radioactivity catastrophes in the past years. Such as, the Chernobyl nuclear plant in Ukraine, Flixborough United Kingdom Explosion in 1974, and Fukushima Japan, in 2011, which destroyed power utilities worth billions of dollars, caused unnecessary deaths, and posed a lasting effect on the immediate environment [1]. Another outstanding issue affecting the nuclear power sector today is the problem of decommissioning the stations to the point that no radioactive treats is posed [2, 3]. For these facts therefore, a two arm mobile manipulator delivery platform Multi-Arm Robot System for Nuclear Decommissioning (MARS-ND) called HYDROLEK HLK-7W, is developed at Lancaster University in order to achieve decommissioning tasks, repairs and maintenance of nuclear plants.

Hydrolek is a two arm hydraulically actuated manipulator with 7DOF, six rotary joints and one gripper attached to a platform Brokk40 as shown Fig. 1. The very first attempts in using MARS-ND platform for decommissioning applications are reported in [4] and [5] in which forward and inverse kinematics were utilized in order to model the motions of the robot. Subsequently linear PID and PIP

Allahyar Montazeri is with Engineering Department, Lancaster University Gillow Avenue, Bailrigg, LA14YW (corresponding author: 00441524 593660; e-mail: a.montazeri@ lancaster.ac.uk).

Josef Ekotuyo is with with Engineering Department, Lancaster University Gillow Avenue, Bailrigg, LA14YW (e-mail: e.udo@lancaster.ac.uk). control algorithms were designed, implemented, and tested enabling the robot to be controlled and operated with some degrees of autonomy [6]. To overcome the effects of slow and imprecise control due to the nonlinearities of the hydraulically-driven actuators another control approach for the Hydrolek manipulator proposed in [8] relying on a nonminimal state variable feedback techniques. More investigation on suitability of state dependent parameter (SDP) control approach was carried out in [9] by demonstrating a better desirable joint motion using the SDP control technique. However, in all of these results the model adopted for controller design is obtained experimentally by collecting input/output data for each joint. An experimental model for the hydraulically actuated mobile dualmanipulators based on state dependent system identification technique is proposed in [10] and applied for control design purpose in [11].

Although the above mentioned control techniques proved to be useful for independent joint control and overcome mild nonlinearities for a limited operating conditions of the HYDROLEK arm, they cannot be yet applied to handle control tasks in more realistic decommissioning missions. More specifically, task patterns in nuclear reactor operational environment requires reliable control algorithms which will be able to provide an estimation that maintains predictive power both in mild, high speed and difficult tasks carried out by the manipulator. This necessitates development of more sophisticated and high performance identification [7, 15, 16, $22,23,24]$ and model-based control algorithms working based on a reliable dynamic model of the manipulator. Therefore, developing a more general nonlinear dynamic model of the bespoke robot which has the potential to be utilized for design and development of such a model-based nonlinear control strategies determines the major motivation of this paper. This will result in a general nonlinear simulator for the bespoke manipulator with the capability of tuning its parameters to generate the outputs similar to the experimental data gathered from the real manipulator under different operating conditions. The developed model can be also used for optimal excitation trajectory design and identification of parameters of the manipulator as explained in $[12,13$, and 14]. This in turn facilitates determination of parametric and non-parametric uncertainties of the dynamic model of the robot shown in Fig. 1.

By integrating the dynamic model of different subsystems of the Hydrolek arm shown in the schematic block diagram 
of Fig. 2, the aim of this paper is to develop a reliable simulator by which the dynamic and kinematic characteristics of the 7DOF hydraulically actuated teleoperated robot platform shown in Fig. 1 can be derived. This is especially essential for design and implementation of different joint-level and supervisory control algorithms to accomplish decommissioning tasks semi-autonomously and by considering the remote operator in the loop. The approach adopted here is to investigate the modelling of various components of the manipulator using symbolic, numerical and experimental techniques and then integrate them in a unified Simulink model for the purpose of system identification, parameter estimation, design of trajectories as well as control objectives. This is usually referred to as Robot Calibration in robotic terminologies [17].

One of the important features of the tele-operated robots used for decommissioning applications is that they should be rug enough to enter into the hostile and unstructured environments and handle, crash, or cut heavy objects. This necessitates the use of nonlinear hydraulic actuators in joint constructions of such robotic platforms. Looking at the literature $[18,19,20,21]$ reveals that due to difficulties in modeling and control of hydraulically actuated manipulators they are not investigated as often as motor-driven joints. Our approach in modeling is to use the CAD model of the $\rightarrow$ HYDROLEK arm along with the SimMechanic Toolbox for mechanical subsystem and then integrate this with the model hydraulic actuator developed for each joint using SimScape Toolbox. Later the parameters of the model such as joint stiffness, and joint damping are tuned based on the measured experimental results and defining a proper fitness function.

On these notes the paper is organized as follows. In section II, symbolic modeling of Hydrolek is developed by writing first principle mechanical, hydraulic and magnetism law applied to each subsystem of the manipulator. A numerical model is subsequently implemented in Section III using both dedicated Simulink toolboxes and symbolic dynamics equation defined earlier in the symbolic part. Since it is important to verify the numerical model developed for each subsystem validation is done in Section IV using the experimental data. Finally, Section V concludes the paper.

\section{SYMBOLIC MODELING}

A more detailed block diagram of the system representing the components of MARS-ND is shown in Fig. 3. By having a high-level description of the system the block diagram can be divided into three main parts: 1- Labview software on remote operator's computer, 2- Brokk base system powered by an electrical power supply, 3 - Two HydroLek arms with the same configurations.

\section{A. Actuating Subsystem}

The components of the actuating subsystem are shown in Fig. 4. This includes the valve system and double acting

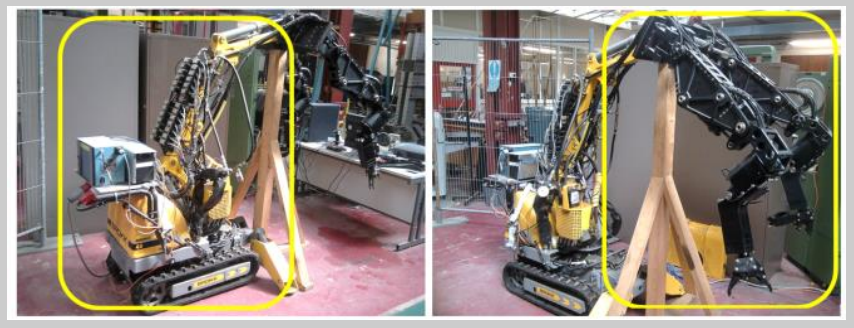

Figure 1. Multi-Arm Robot Platform: Brokk40 base machine (left) and two HydroLek arms (right).

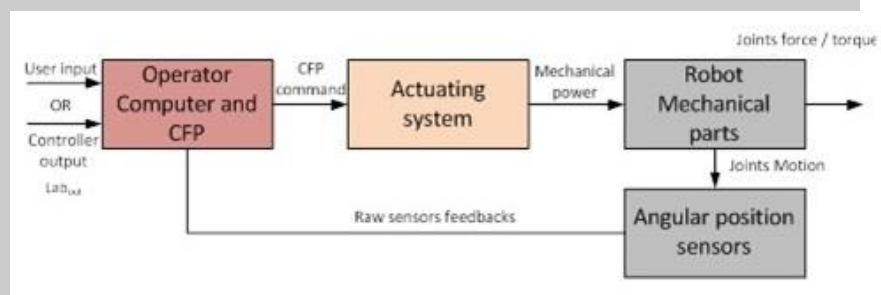

Figure 2. Block diagram of the system adopted for dynamic modeling of the manipulator.

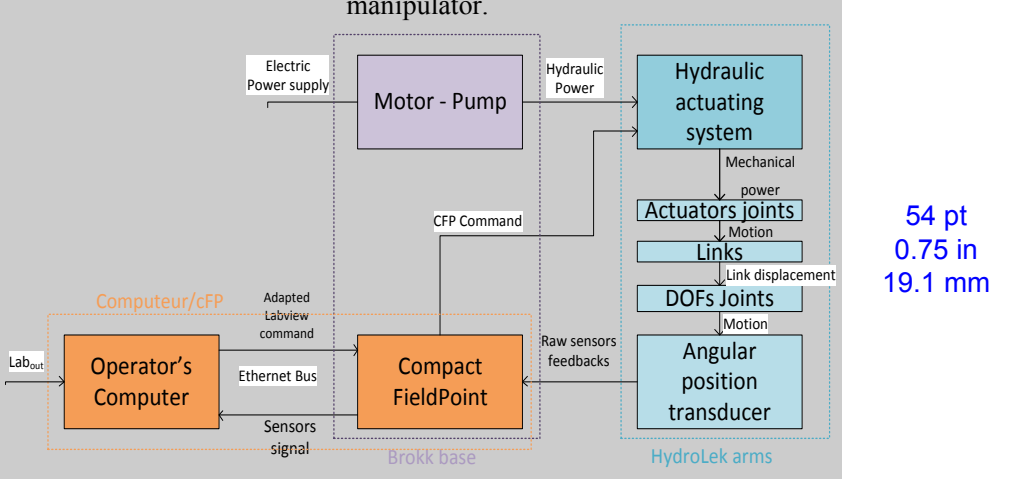

Figure 3. High level description of MARS-ND system.

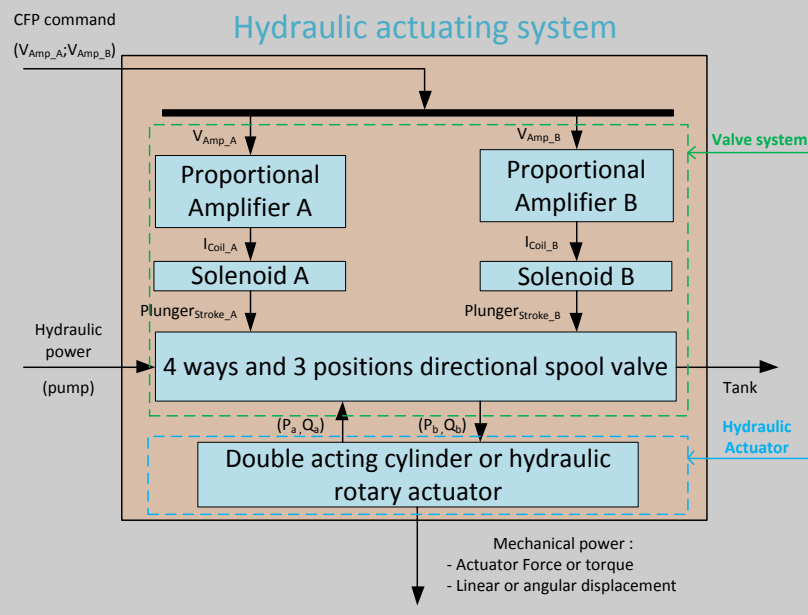

Figure 4. Detailed block of the joint actuating system i.e. hydraulic actuating system.

cylinder. The valve system itself includes amplifier, solenoid, and spool directional valve blocks as illustrated in 
Fig. 4. By writing the input-output relations for each block the dynamic model of the actuating subsystem can be derived.

\section{B. Mechanical Subsystem}

Mechanical subsystem involves actuator joints, links and DOF joints blocks as shown in Fig. 3. The input of the whole mechanical subsystem is the actuating torque applied to each joint and its output is the angular position of each joint. Actually the angular position of the first five joints are the only variables that can be sensed on the real robot. To be able to model this subsystem first of all derivation of symbolic forward dynamics that relates the generated torque as a result of motion of link is required. However, it is possible to adopt some simplification assumptions and consider the HydroLek arm as a serial link. For this purpose the mechanical dynamic of hydraulic actuators is ignored in front of dynamics of the links and their effect which is generating torque at each joint will be only considered. All links are considered infinitely rigid and all joints are assumed to be backlash-free with no friction between them. For the HydroLek arm under the study the position of the origin of the frame of joint $O_{i}$ can be determined based on the available dimensions of links given in CAD model listed $\rightarrow$ in Table 1. Different traditional method such as homogeneous matrix approaches can be used to define the frame orientation. However, a simple rotation matrix $R_{h}(\theta)_{i-1}^{i}$ of angle $\theta$ and around the axis $h$ can be used between joint $i$ and $i-1$ to define the orientation of a frame. Given that all joints are revolute, each link $i$ will rotate at a specific angular velocity around the unitary axis of the frame of that joint, i.e. $\left(x_{i}\right.$ or $y_{i}$ or $\left.z_{i}\right)$. The torque and the force vectors, i.e. $\overrightarrow{F_{J_{l}}}$ and $\overrightarrow{M_{J_{l}}}$, applied at $O_{i}$ for each joint $i$ is expressed in terms of the fixed frame $F_{0}$. Figure 5 shows the schematic of link $i$ and its corresponding parameters. For each link except the last one, the force balance equations can be written at point $G_{i}$ in terms of the base frame $F_{0}$ using recursive Newton Euler approach. As the last link is of rotary form a different setting is used for derivation of dynamics of that joint. Writing the force and momentum balance equations for all links leads to a total of 36 equations ( 6 per each insulated link). Then, the system of equations should be solved having torques and forces of joints unknown.

TABLE 1. LINK DIMENSIONS IN MM.

\begin{tabular}{cccc}
\hline Link $_{\boldsymbol{i}}$ & $\boldsymbol{L}_{\boldsymbol{i}} \boldsymbol{X}_{\boldsymbol{i}}$ & $\boldsymbol{L}_{\boldsymbol{i}} \boldsymbol{Y}_{\boldsymbol{i}}$ & $\boldsymbol{L}_{\boldsymbol{i}} \boldsymbol{Z}_{\boldsymbol{i}}$ \\
\hline $\mathbf{0}$ & 80 & 0 & 0 \\
$\mathbf{1}$ & 137.29 & 35.36 & -32.01 \\
$\mathbf{2}$ & 413.25 & 301.53 & 0 \\
$\mathbf{3}$ & 161 & -84.85 & 0 \\
$\mathbf{4}$ & 44.45 & -129.3 & 0 \\
$\mathbf{5}$ & 18.45 & 278.05 & 0 \\
$\mathbf{6}$ & 150 & 0 & 0 \\
\hline
\end{tabular}

The result is the expression of joints torques and forces in the fixed base frame. As solving such a system of equations is really difficult by hand Matlab symbolic Toolbox and Mupad software has been used to set the robot configuration parameters and then an automatic symbolic equation solver of Mupad will derive the required forces and momentums. The written code has about 800 lines and is designed to solve for the forward dynamic of all kinds of serial robots with prismatic and/or revolute joints. To derive the kinematic and dynamic characteristics it is enough just to define a complete robot configuration and then execute the Mupad notebook. A graphical user interface GUI is designed (about 2000 code lines) in order to have a more convenient way to describe robot configuration, calculate the symbolic dynamics and kinematics model and implement dynamics and kinematics numerical model in Simulink. The flow diagram of different components of the developed software for symbolic modeling is depicted in Fig. 7.

\section{Sensing Subsystem}

Each of five first joints on each arm are fitted with rotary potentiometer sensors to record the joints angular motion. A common linear relation is assumed between joint angle input and the output voltage signal of joint. Thus, the model of the sensing subsystem for each joint $i$ shown in Fig. 3 can be represented by this linear equation

$$
\text { Angle }_{\text {joint }}\left(\text { Sensor }_{\text {RawData }}\right)=A_{i} \text { Sensor }_{\text {RawData }_{i}}+B_{i}
$$

where $A_{i}$ and $B_{\mathrm{i}}$ are constant coefficients of the $i$ th potentiometer and should be tuned for each joint in a calibration process.

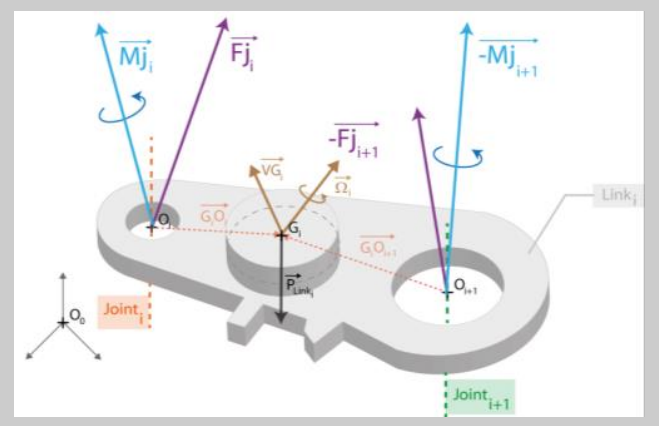

Figure 5. Insulation of links (not true for last link).

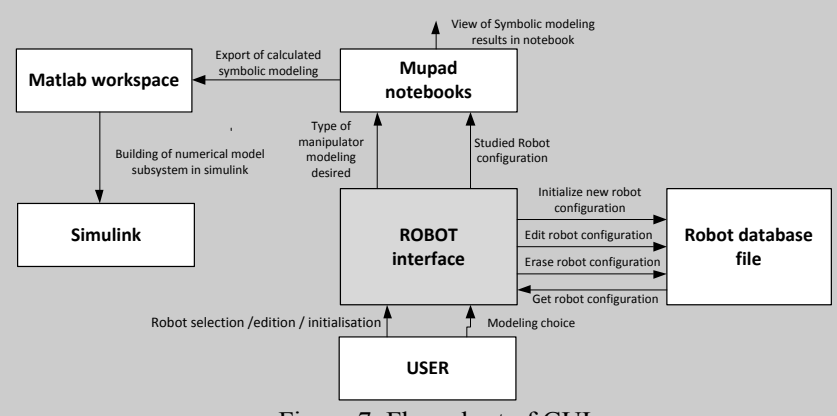

Figure 7. Flow chart of GUI. 


\section{NUMERICAL MODELING}

Referring to Fig. 2 we intend to find a numerical model of the block diagram of the system shown in this figure. Despite of actuating subsystem, mechanical subsystem, and sensing subsystems the operator/CFP block will be also modeled here to manage the flow of signals in a more realist way. This block in the real system is implemented using LabVIEW software and is modeled here numerically with a block called LabVIEW adaptation module. It was developed using basic Simulink blocks such as arithmetic and IF conditional blocks. IF conditional block will activate the desired output by analyzing the sign of $\mathrm{Lab}_{\text {out }}$ input to actuate amps $A$ or $B$.

\section{A. Actuating Subsystem}

The model of the actuating subsystem is developed by using blocks from standard Simulink library and built-in hydraulic and electrical components available in SimHydraulics and SimElectronics toolboxes. Actuating subsystem consists of two subsystems itself: 1- Valve subsystem, 2- Hydraulic cylinders. Valve system is decomposed into proportional amplifier, solenoid valve and directional valve. Numerical model of all of these components implemented in Simulink are shown Figs. 8, 9, and 10 respectively. Hydraulic cylinder block contains numerical model of hydraulic joint actuator, i.e. double acting cylinder or hydraulic rotary actuator model (depending on the type of the joint). The real system operates with double acting cylinder and hydraulic rotary actuator for the last two joints. Built-in blocks available in SimHydraulics library are used to model the two stated types of hydraulic actuator. The inputs of hydraulic cylinder block are two hydraulic ports $A$ and $B$ coming from output of the valve system. Output is the mechanical port $R$ or $S$ corresponding to the mechanical rod or actuator shaft of the hydraulic cylinder. These are shown in Fig. 10. Hydraulic power source block contains a hydraulic source output and a tank input port for valve system. To develop a basic model of the pump it is considered as an ideal pressure source where pressure setting is specified with a constant Simulink block named pressure pump. Custom hydraulic fluid block specifies charactetistics of the fluid used in HydroLek system.

\section{B. Mechanical Subsystem}

The model is built by using common Simulink blocks and the blocks available in SimMechanics Toolbox. The blocks used for modeling of the mechanical subsystem of the robot can be categorized into the following sections: 1- simulation configuration blocks, 2- the blocks used to define the joint types, 3- the blocks used to determine the position and orientation of the frames, 4 - the blocks used to determine the links, and 5- utility blocks. The goal of simulation configuration block is to arrange the base fixed frame, position of the gravity vector and configure the solver parameters of the model. To determine the position and orientation of the joints frames the rigid transformation block is used here. There are different methods to define the transformation matrix, however, here a Cartesian transformation matrix is chosen to change the properties of the output frame with respect to the base frame.

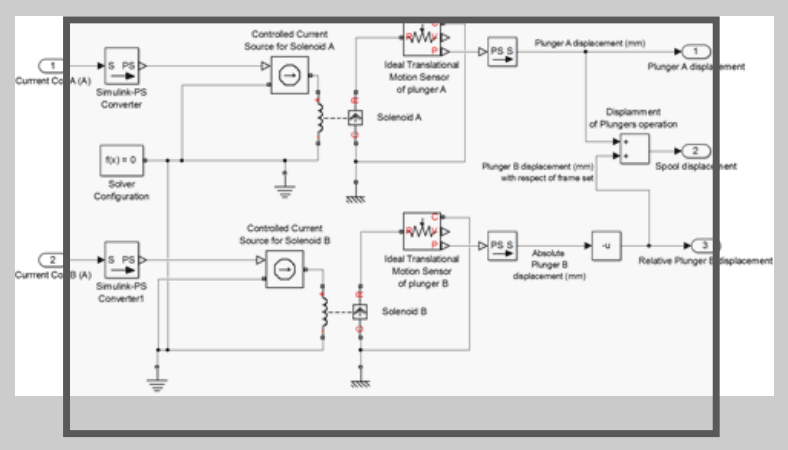

Figure 9. Numerical model of the proportional solenoid valve.

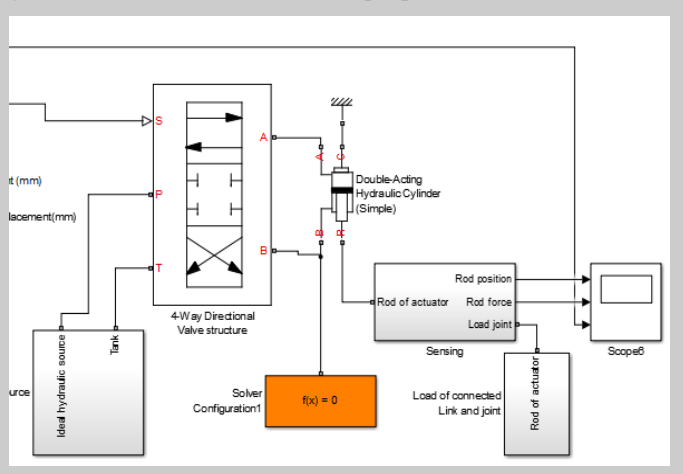

Figure 10. Numerical model of the directional valve.

SimMechanics Toolbox contains a library of different joint types. To configure revolute joints in this study, as shown in Fig. 11, the base frame must be connected to the port $B$ of the block. The output frame at port $F$ is a basis named follower and can represent for example the rotation around the $z$ axis. In order to have a complete description of the manipulator link, several other parameters need to be specified. These parameters are set by a block in SimMechanics toolbox called Solid. . As can be seen in Fig. 12 the geometrical properties of the links, i.e. the shape and dimension of the links, are all saved in a shape file exported from the Solidwork software in STL file. Other parameters of the link can also be defined within this block. The developed model takes into account both inertia parameters of each link and energy dissipation at each revolute joint. Inverse and forward dynamics derivation can be studied easily by changing just a few model parameters, i.e. joint actuation modes and signal converter parameters. A 3D representation of the manipulator implemented numerically 
by Simulink blocks is shown in Fig. 13 with the help of the Mechanical Explorer in Simulink.

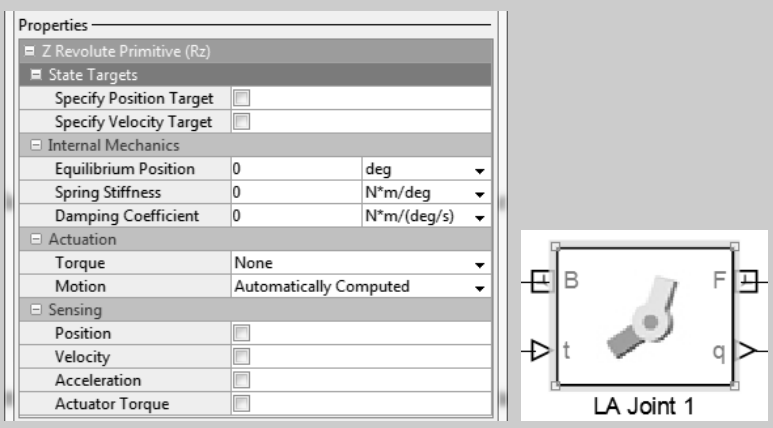

Figure 11. Configuration block of the revolute joint (left) icon of SimMechanics block (right).
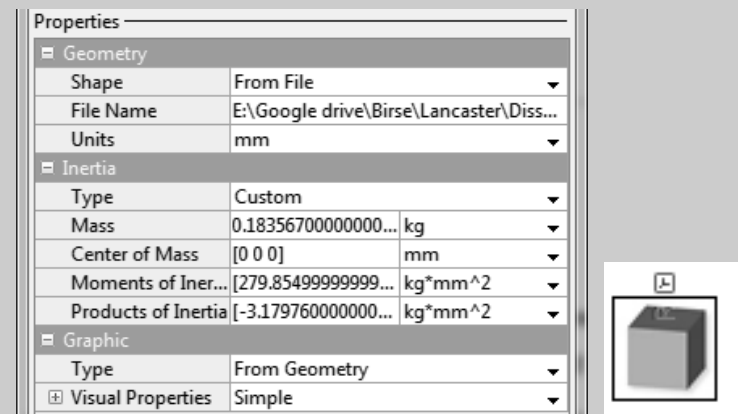

Figure 12. Configuration block of the Solid block (left), icon of the Solid block (right).

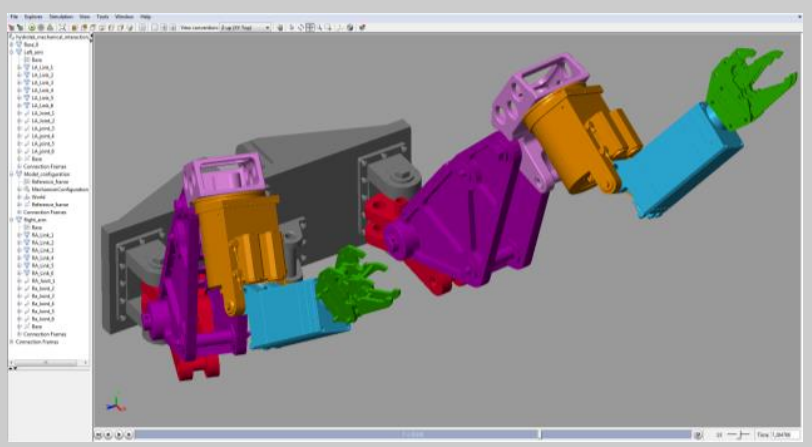

Figure 13. A 3D view of the developed dual-arm HydroLek manipulator shown in the Mechanical Explorer.

\section{MOdEL TUNING AND VALIDATION}

To validate the developed model in the previous sections and evaluate whether it represents the real robot a series of input and output experimental data are collected. Due to lack of space the results for joint two are only presented here. The input/output experimental data are gathered by doing experiment on the Hydrolek manipulator shown in Fig. 1. The input data is the voltage applied to the cFP subsystem in Fig. 2 and the output data is the angle of rotation for joint two measured using potentiometers installed on each joint. These input/output data, shown in Fig. 14, are used for validation of the Simulink model as the reference data and are compared with the numerical results achieved from the developed Simulink model. As can be seen from Fig. 14, the input signal is a multi-level PRBS voltage signal exciting joint 2 of the robot, while the other inputs are set to be zero. Studying the differences between the output of the numerical model and the experimental results aid for better understanding of discrepancies between the dynamics included in the Simulink model and the real robot. To this end, the parameters of each of mechanical and actuating subsystems shown in Fig. 2 are tuned separately. After several try and error and it turned out the following parameters are the most important ones affecting the behavior of the output of the model substantially: joint stiffness, and parameter of the double acting cylinder. The performance index used to compare the results is called normalized root mean square error (NRMSE) and calculated as follows

$$
\text { Fit }(i)=1-\frac{\|x r e f(:, i)-x(:, i)\|}{\|\operatorname{xref}(:, i)-\operatorname{mean}(\operatorname{xref}(:, i))\|}
$$

After fine tune of the above mentioned parameters using NRMSE the output shown in Fig. 15 is plotted against the measured experimental data. This validate the creditability of the developed model, however, it is expected more advanced approach on optimization of the parameters of the model results in better outputs.
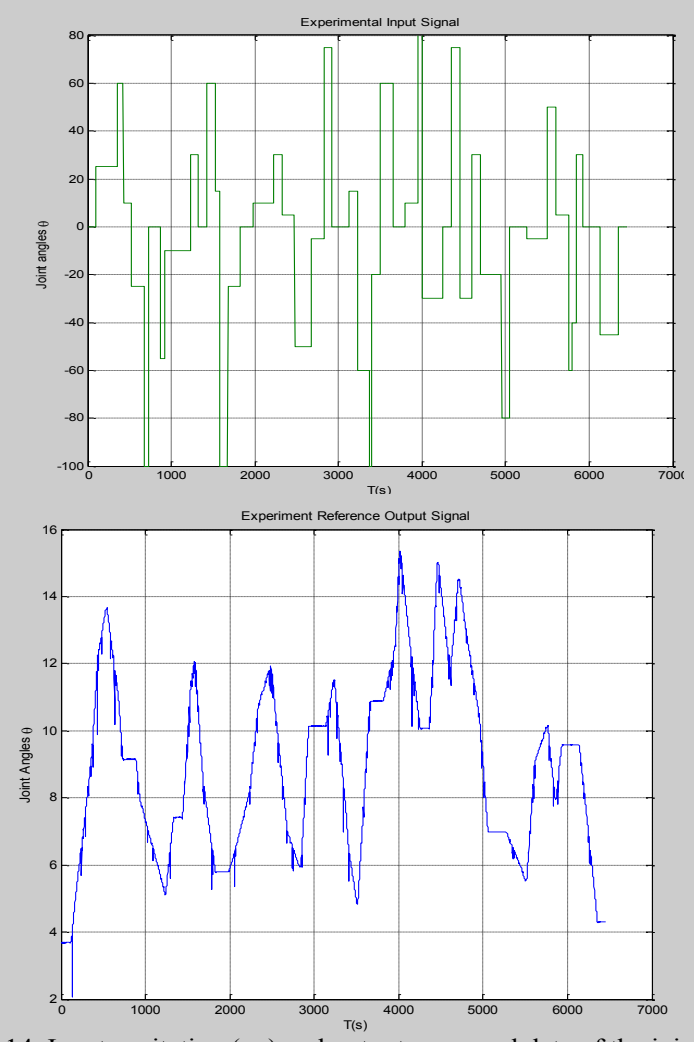

Figure 14. Input excitation (up) and output measured data of the joint two.
$54 \mathrm{pt}$ 0.75 in $19.1 \mathrm{~mm}$ 


\section{$54 \mathrm{pt}$} 0.75 in $19.1 \mathrm{~mm}$

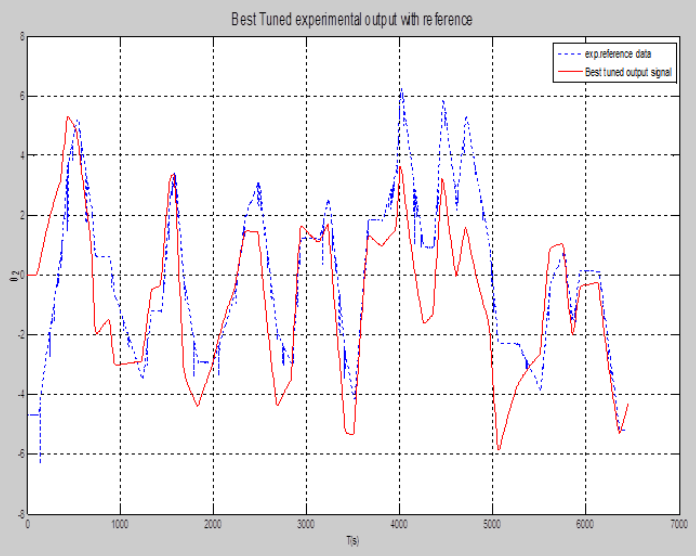

Figure 15. Comparison of the model output and experimental for joint two.

\section{CONCLUSION}

In this paper a comprehensive model of the 7DOF hydraulically actuated tele-operated robot called HydroLek manipulator is developed and validated using symbolic and numerical modeling techniques. To make sure the model generated the results comparable to the real arm it is validated by several experiments and then the parameters of the model are tuned to reduce the error fitness function. The model can be used to derive dynamic and kinematic characteristics of the robot. These results will be used later for optimal trajectory design, system identification, and controller design in joint and Cartesian spaces. Since the robot is going to be deployed for decommissioning tasks precise manipulation and handling of the objects by cooperation of the model-based intelligent control system and the remote operator is of particular importance.

\section{REFERENCES}

[1] J. Samseth, A. Banford, B. Batandjieva-Metcalf, M. C. Cantone, P. Lietava, H. Peimani and A. Szilagyi, "Closing and Decommissioning Nuclear Power Reactors: Another Look Following the Fukushima Accident," United Nation Environment Program, Year Book, 2012.

[2] R. Bloss, "How do you decommission a nuclear installation? Call in the robots," International Journal of Industrial Robot, vol. 37, pp. 133-136, 2010.

[3] R. Bogue, "Robots in the Nuclear Industry: a Review of Technologies and Applications," International Journal of Industrial Robot, vol. 38, pp. 113-118, 2011.

[4] D.W. Seward, The Development of Intelligent Mobile Robots. PhD Thesis. Lancaster University, 1999, U.K.

[5] M.J. Bakari, K.M. Zeid, and D.W. Seward, "Development of a Multiarm mobile Robot for Nuclear Decommissioning Tasks," International Journal of Advanded Robotics Systems, vol. 4, pp. 384406, 2007.

[6] E.M. Shaban, S. Ako, C.J. Taylor, D.W. Seward, "Development of an Automated Verticality Alignment System for a Vibro-lance," Automation in Construction, vol. 17, No. 5, pp. 645-655, 2008.

[7] S. Mahmoodi, A. Montazeri, J. Poshtan, M.R. Jahed-Motlagh, M. Poshtan, "Volterra-Laguerre modeling for NMPC," 9th IEEE International Symposium on Signal Processing and Its Applications, ISSPA 2007, pp. 1-4, 2007.
[8] E.M. Shaban, K. Zied, C.J. Taylor, D.W. Seward, "Nonlinear Control System Design for Construction Robots Using State-Dependent Parameter Models," 22th International Symposium on Automation and Robotics in Construction, Italy, Sept. 2005.

[9] C.J Taylor, A. Chotai, D. Robertson "State-Dependent Control of a Robotic Manipulator Used for Nuclear Decommissioning Activities," IEEE International Conference on intelligent Robots and System (IROS-10), vol. 239, pp. 2413-2418, October 2010.

[10] D. Robertson, C. J. Taylor , C. Lokuciewski, "State-Dependent System Identification for Control of a Hydraulically-Actuated Nuclear Decommissioning Robot," 16th IFAC Symposium on System Identification. ed. / Michel Kinnaert. Brussels: IFAC, pp. 759-764, 2012.

[11] C.J. Taylor, D. Robertson, "State-dependent control of a hydraulically actuated nuclear decommissioning robot," Control Engineering Practice, vol. 12, no. 21, pp. 1716-1725, December 2013.

[12] J. Swevers, C, Ganseman, D.B. Tukel, J. De Schutter, "Optimal Robot Excitation and Identification," IEEE Transactions on Robotics and Automation, vol. 13, no. 5, pp. 730-740, 1997.

[13] X. Liao, W. Wang, Y. Lin, C. Gong, "Time-optimal trajectory planning for a $6 \mathrm{R}$ jointed welding robot using adaptive genetic algorithms," International Conference on Computer, Mechatronics, Control and Electronic Engineering, vol. 2, 2010.

[14] J. Swevers, C. Ganseman, J. De Schutter, H. Van Brussel, "Experimental Robot Identification Using Optimized Periodic Trajectories," Mechanical Systems and Signal Processing, vol. 10, no. 5, pp. 561-577, 1996.

[15] M.M. Arefi, A. Montazeri, J. Poshtan, M.R. Jahed-Motlagh, "Nonlinear model predictive control of chemical processes with a Wiener identification approach," IEEE International Conference on Industrial Technology, ICIT 2006, pp. 1735-1740, Mumbai, India 2006.

[16] E. Villagrossi, N. Pedrocchi, F. Vicentini, L.M. Tosatti, "Optima Robot Dynamics Local Identification using Genetic-based Path Planning in Workspace Subregions," IEEE/ASME International Conference on Advanced Intelligent Mechatronics (AIM), pp. 932937, 2013.

[17] R. Berhhardt. Robot Calibration. Chapman \& Hall, London, UK 1993.

[18] J. Heintze, G. van Schothorst, A. J. J. v.d. Weiden, and P. C Teerhuis, "Modeling and Control of an Industrial Hydraulic Rotary Vane Actuator," in Proceedings of the 32nd IEEE Conference on Decision and Control, pp. 1913-1918, December 1993.

[19] J.E. Bobrow, K. Lum, "Adaptive, High Bandwidth Control of a Hydraulic Actuator," ASME Journal of Dynamic Systems, Measurement and Control, vol. 118, no. 4, pp. 714-720, 1996.

[20] C.G. Zhejiang, S. Pan, "Nonlinear Adaptive Robust Control of SingleRod Electro-Hydraulic Actuator with Unknown Nonlinear Parameters," IEEE Transactions on Control Systems Technology, vol 16, no. 3, pp. $434-445,2008$.

[21] J. Yao, Z. Jiao, D. Ma, and L. Yan, "High-Accuracy Tracking Control of Hydraulic Rotary Actuators with Modelling Uncertainties," IEEE/ASME Transactions on Mechatronics, vol. 19, no. 2, pp. 633641, 2014.

[22] H. Esmaeilsabzali, A. Montazeri, J. Poshtan, M.R JahedMotlagh, "Robust identification of a lightly damped flexible beam using setmembership and model error modeling techniques," IEEE International Conference on Control Applications, pp. 2945-2949, Munich, Germany, Oct. 2006.

[23] S. Ahmadizadeh, A. Montazeri, J. Poshtan, "Identification of a stable model for a flexible plate using frequency domain subspace methods," IFAC World Congress, vol. 18, no. 1, 6517-6522, 2011.

[24] A. Montazeri, H. Esmaeilsabzali, J. Poshtan, M.R. JahedMotlagh, "Comparison of different approaches for robust identification of a lightly damped flexible beam," 14th European Signal Processing Conference, pp. 1-5, 2006. 